\title{
The Role of Gonadal Hormones in Neuromuscular Synapse Elimination in Rats. II. Multiple Innervation Persists in the Adult Levator Ani Muscle After Juvenile Androgen Treatment
}

\author{
Cynthia L. Jordan, Michael S. Letinsky, ${ }^{1}$ and Arthur P. Arnold \\ Department of Psychology and Laboratory of Endocrinology, Brain Research Institute, University of California, Los \\ Angeles, Los Angeles, California 90024, and 'Department of Physiology and Ahmanson Laboratory of Neurobiology, \\ University of California, Los Angeles, Los Angeles, California 90024
}

In the previous study (Jordan et al., 1989), we demonstrated that androgen treatment of juvenile male rats inhibits the elimination of synapses in the levator ani (LA) muscle. In the present study, we asked whether synapse elimination would occur once this juvenile androgen treatment ended. Castrated male rats were given androgen during a juvenile treatment period (7-34 d) and were killed 4 or 8 weeks after the end of androgen treatment (at 9 or 13 weeks after birth). The adult pattern of innervation in the LA was assessed (1) anatomically by counting the number of stained motor axons innervating single muscle fibers and (2) electrophysiologically by counting the number of components in intracellularly recorded endplate potentials. Based on the number of stained motor axons, the LA from juvenile androgen-treated castrates had as much multiple innervation 1 and 2 months after the end of androgen treatment (at 9 and 13 weeks) as was present during androgen treatment at 4 weeks. This suggests that no further synapse loss occurred in the LA once androgen treatment ended. Based on electrophysiological measures, adult LA muscles previously exposed to androgen were found to contain significantly more polyneuronal innervation than normal adult LA muscles. Juvenile androgen treatment also increased the size but not the number of motoneurons in the spinal nucleus of the bulbocavernosus which contains LA motoneurons. Thus, the increased level of multiple innervation in the LA is not due to a higher than normal number of motoneurons innervating this muscle. Because multiple innervation persists in the LA in the absence of continued androgen treatment, androgen may have permanently prevented synapse elimination. This observation suggests that a similar hormonal regulation of synapse elimination may contribute to the development of adult sex differences in the synaptic organization of the brain.

During development, steroid hormones are known to have permanent effects on the structural and functional organization of the adult nervous system (Arnold and Gorski, 1984; Arai et al., 1986; Arnold and Jordan, 1988; Fishman and Breedlove, 1988).

\footnotetext{
Received Apr. 1, 1988; revised June 9, 1988; accepted June 29, 1988.

We wish to thank Dr. Alan Grinnell for his comments on the manuscript and Jean Lee and Tony Alexander for their technical assistance. This work was supported by USPHS Grants HD15021, NS13470, and HD7228.

Correspondence should be addressed to Cynthia L. Jordan, Department of Psychology, University of California, Los Angeles, Los Angeles, CA 90024-1563. Copyright (C) 1989 Society for Neuroscience $0270-6474 / 89 / 010239-09 \$ 02.00 / 0$
}

Such effects can involve changes in the number and size of adult neurons (Gurney, 1981; Jacobson et al., 1981; Breedlove and Arnold, 1983a, b; Nordeen et al., 1987), as well as in the number and specificity of synaptic connections between adult neurons and their targets (Raisman and Field, 1973; Nishizuka and Arai, 1981a, b; Breedlove, 1985; Matsumoto and Arai, 1986b). For example, gonadal androgen during a perinatal period of development irreversibly increases the size and number of adult motoneurons in the sexually dimorphic spinal nucleus of the bulbocavernosus (SNB) (Breedlove and Arnold, 1983b). Until recently, however, it has not been clear which cellular processes in development are influenced by steroid hormones. For example, hormones might alter the adult number of neurons by influcncing the birth and/or survival of ncurons. Recent evidence suggests that gonadal steroid hormones are capable of regulating such developmental processes as cell proliferation (Sassoon et al., 1986), neurite outgrowth (Toran-Allerand, 1976), cell death (Breedlove, 1984; Bennett and Truman, 1985; Nordeen et al., 1985) and synaptogenesis (Matsumoto and Arai, 1979; Nishizuka and Arai, 1982; DeVoogd et al., 1985; Matsumoto et al., 1988a). In this report, we evaluate the question of whether gonadal hormones might also permanently influence the adult organization of the nervous system by regulating the normal ontogenetic process of synapse elimination.

Synapse elimination occurs in many different parts of the developing nervous system, including developing neuromuscular systems (Purves and Lichtman, 1980; Thompson, 1986; Betz, 1987). During synapse elimination in muscle, a neonatal pattern of multiple innervation of single muscle fibers is converted to the adult pattern of single innervation through a selective elimination of excess synaptic connections (Redfern, 1970; Brown et al., 1976; Riley, 1977). While synapse elimination occurs in most rat muscles around the first 2 weeks after birth (Van Essen, 1982), this process occurs substantially later in the androgen-sensitive levator ani (LA) muscle of the rat (Jordan et al., 1988a). In a previous study, we provided evidence that treatment of rats with androgen during juvenile life delays synapse elimination in the LA but not in the extensor digitorum longus (EDL) (Jordan et al., 1989): In the present study, we asked whether synapse elimination would occur once the juvenile treatment ended.

In this study, we addressed this question by assessing the pattern of innervation in the LA at 1 and 2 months after the end of juvenile androgen treatment. Both histological and electrophysiological techniques were used to assess the pattern of 
innervation in the LA. We also measured the size and number of motoneurons in the SNB, which contain motoneurons that innervate the LA (Breedlove and Arnold, 1980; Schroder, 1980). We find that a high level of anatomical multiple innervation is maintained in the LA for at least 2 months after the end of juvenile androgen treatment and that such muscles show more functional polyneuronal innervation than normal.

\section{Materials and Methods}

Surgical and treatment procedures. All animals used in this study were Sprague-Dawley male rats obtained from Simonsen Laboratories or derived from pregnant dams obtained from this supplier. Procedures used for castrating and treating rats were the same as previously described (Jordan et al., 1989). In brief, rats were castrated on postnatal day (P) 7 and given daily injections of either testosterone propionate (TP) $(100 \mu \mathrm{g} \mathrm{TP} / 50 \mathrm{gm}$ body weight) or the vehicle oil for a period of 4 weeks, between P7 and P34. There were 2 additional control groups: normal untreated males and males that were sham castrated on P7 and given daily injections of oil during the same treatment period (P7-34). Animals were weaned at P21 and housed as litters in group cages until they were killed at 9 or 13 weeks, after an additional 4 or 8 weeks without treatment.

Histological procedures. At the time of death, animals were overdosed with sodium pentobarbitol. Their LA and EDL muscles were dissected, stained with tetranitroblue tetrazolium (TNBT), and prepared for microscopy as previously described (Jordan et al., 1988a). The unperfused lumbar/sacral portion of the spinal cord was also removed from some of these animals and was put in a $0.135 \mathrm{~m}$ sodium phosphate-buffered solution (pH 7.2) containing $10 \%$ formalin. After several weeks of formalin tixation, spinal cords were frozen and sectioned transversely at $50 \mu \mathrm{m}$ thickness using a sliding microtome. Alternate spinal cord sections were mounted on coated slides and stained with either thionin or cresyl violet.

Microscopic analysis. LA and EDL muscles and spinal cords obtained from 4-6 animals from at least 2 different litters per group were analyzed without knowledge of treatment conditions. The procedure used for analyzing TNBT-stained muscles was the same as previously described (Jordan et al., 1989) and involved measuring for each sampled muscle fiber the number of different axons and preterminal branches from the same axon that contacted the fiber, the number of junctional sites formed by such axons on a fiber, and, using a calibrated ocular micrometer, the distance between junctional sites on a fiber and the width of muscle fibers. Some of the data obtained from normal males has previously been reported in a related study (Jordan et al., 1988a).

The size of SNB motoneurons is permanently increased by androgen secretions just before and after birth, and is also regulated by androgen in adulthood (Breedlove and Arnold, 1981, 1983b; Kurz et al., 1986; Forger and Breedlove, 1987; Lee et al., 1988). For this reason, the spinal cords from juvenile androgen- or oil-treated castrates at 9 weeks were compared with another control group of 9-week-old males castrated at P35 (the time at which treatment ended for males castrated on P7). Thus, all 3 groups were without gonadal androgen during the 4 weeks prior to death. Such late-castrated males were left untreated during the juvenile treatment period (P7-34). Comparable procedures were used for sacrificing, removing, and processing the spinal cords of late-castrated males. Based on alternate cross sections of the lumbar spinal cord, the number of large, densely staining neurons that had distinct nuclei were counted in the region of the SNB. Such motoneurons are located in the dorsomedial aspect of the ventral horn in lumbar segments 5 and 6 (Breedlove and Arnold, 1981). Counts of SNB motoneurons were corrected according to Konigsmark (1970) as previously described (Breedlove and Arnold, 1981). Between 25 and 30 SNB cells were also chosen at random throughout the rostrocaudal extent of the nucleus for measuring the cross-sectional area of their somata and nuclei using a digitizing tablet interfaced with a computer.

Data obtained from muscles were statistically analyzed using an analysis of variance (ANOVA) design (Keppel, 1982) and as previously described (Jordan et al., 1989). Two-way ANOVAs (treatment $x$ age) were carried out unless otherwise specified. Data on the number and size of SNB motoneurons were statistically analyzed using independent 2-tailed $t$ tests.

Electrophysiological procedures. Multiple innervation was also assessed electrophysiologically in LA muscles from juvenile androgen- treated and normal adult males which did not contribute to the histological data. For these experiments, animals were castrated and treated daily with TP during the 4 week juvenile treatment period (P7-P34) or were left untreated and served as normal control males. Experiments were performed when animals were 9-12 weeks old, following 4-7 weeks without treatment. The LA was rapidly dissected from deeply anesthetized ( $50 \mathrm{mg}$ sodium pentobarbitol $/ \mathrm{kg}$ body weight, i.p.) but respirating animals. At the end of the dissection, animals were given a lethal dose of anesthetic. Dissected LA muscles were pinned as flat whole-mounts in Sylgard-coated dishes that contained oxygenated $\left(95 \% \mathrm{O}_{2} / 5 \% \mathrm{CO}_{2}\right)$ PBS (Card, 1977). During pinning and all subsequent stages of the experiment, LA muscles were constantly perfused with fresh oxygenated PBS. For recording, muscle contractions were blocked by exposure to PBS containing $d$-tubocurarine $\left(6-7 \times 10^{-7} \mathrm{M}\right)$. These concentrations were near threshold for blocking muscle contractions and yielded the largest subthreshold endplate potentials. The number of synaptic inputs to a fiber was determined using standard intracellular recording procedures (Redfern, 1970; Brown et al., 1976; Balice-Gordon and Thompson, 1988), which involved counting the number of discrete reproducible changes in the amplitude of endplate potentials produced by systematically varying the strength and polarity of nerve stimulation. Intracellular recording of resting and endplate potentials were made using $0.6 \mathrm{M} \mathrm{K}_{2} \mathrm{SO}_{4}$-filled glass microelectrodes of $25-50 \mathrm{M} \Omega$ resistance, and the whole LA nerve was stimulated (100 $\mu \mathrm{sec}$ square pulses) using a suction electrode. Hoffman modulation-contrast optics $(400 \times)$ were used to visualize the region of nerve terminals and to facilitate microelectrode placement adjacent to the junctional site. Superficial muscle fibers were sampled across the entire surface of the LA from 5 normal and 4 juvenile androgen-treated castrated males, with 15-65 fibers sampled per muscle (mean, 36 fibers). Muscle fiber resting potentials were generally between -70 and $-80 \mathrm{mV}$. Those fibers with resting potentials more depolarized than $-60 \mathrm{mV}$ were not used. A 1-way ANOVA was used to determine significant differences.

\section{Results}

Pattern of innervation in TNBT-stained $L A$ and EDL muscles Number of axons innervating LA and EDL muscle fibers. Our objective was to determine whether the level of multiple innervation changed after the end of androgen treatment. To accomplish this, we compared the level of multiple innervation in LA muscles 4 and 8 weeks after the end of treatment (at 9 and 13 weeks) with that in the LA at P28, during androgen treatment (data reported in Jordan et al., 1989). In adult ( 9 and 13 week) LA muscle, multiply innervated fibers were those fibers contacted by 2 or more different myelineated axons (Fig. 1). Axons were considered to be different if they were not seen to come from the same parent axon.

In LA muscles from juvenile androgen-treated (JAT) castrates, the incidence of multiply innervated fibers did not change significantly between 4 (P28) and 13 weeks (Fig. $2, p>0.05$ ), indicating that the high level of multiple innervation in androgen-treated I A muscles at 4 weeks was maintained for at least 2 months beyond the end of treatment. At both 9 and 13 weeks, androgen-treated LA muscles had a significantly higher percentage of multiply innervated fibers than normal LA muscles ( $p<0.001$ for comparisons between JAT and normal muscles at 9 and 13 weeks), a difference similar to that previously reported for androgen-treated and normal LA muscles at 4 weeks (Jordan et al., 1989). Moreover, a substantial proportion of the multiple innervation in adult JAT muscles (13-17\% of total muscle fibers) involved 3 or more axons, whereas the multiple innervation in normal adult LA muscles involved only 2 axons.

In contrast to the stable high level of multiple innervation in JAT LA muscles, the level of multiple innervation in the LA from normal males and oil-treated castrates declined between 4 and 13 weeks (Fig. 2). For normal LA muscles, the percentage of multiply innervated fibers remained stable between 4 and 9 

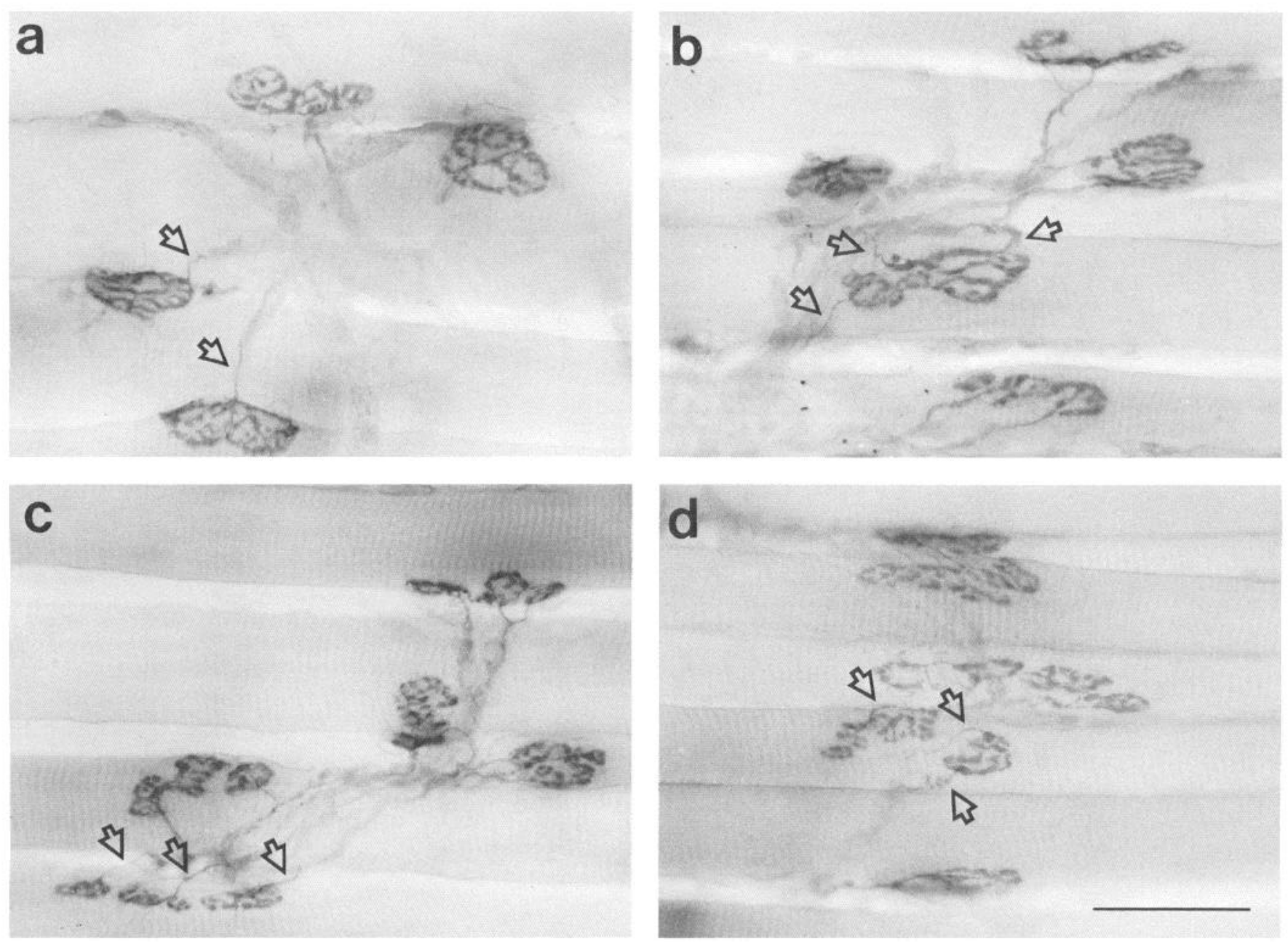

Figure 1. TNBT-stained LA motor nerve terminals in 9-week-old normal males $(a)$ and adult males previously treated with androgen $(b-d)$. $a$, Single motor axons (arrows) contact individual LA muscle fibers to arborize at single synaptic or junctional sites, representing examples of singly innervated fibers. $b$, Three different myelinated motor axons (arrows) contact an individual LA muscle fiber to arborize at a single junctional site. Note that the morphology of the terminal arborization formed by 3 axons $(b)$ is similar to the morphology of the terminal arborizations formed by single axons $(a)$. $c$ and $d$, Examples of multiple innervation that involve multiple junctional sites. In $c$, 3 axons (arrows) contact an individual LA muscle fiber at 3 separate sites, to form distinctly separate, but closely spaced, terminal arborizations. Each site is singly innervated. In $d$, 3 axons (arrows) innervate an individual LA muscle fiber, but in this case, innervation is at only 2 junctional sites. This results in single innervation of one site and multiple innervation of the other. Such multiple innervation that involves 3 or more axons was present only in adult LA muscles previously treated with androgen and not in normal adult LA muscles. Calibration bar, $50 \mu \mathrm{m}$.

weeks $(p>0.05)$ but dropped significantly between 9 and 13 weeks $(p<0.009)$. The LA from juvenile oil-treated castrates experienced a similar loss, although it occurred earlier, between 4 and 9 weeks ( $p=0.08$; Fig. 2). This resulted in LA muscles from oil-treated castrates having significantly less multiple innervation than normal at 9 weeks $(p<0.005)$. In contrast, at 9 weeks the LA muscles from gonadally intact, juvenile oiltreated controls contained the same amount of multiple innervation as normal ( $p>0.05$; Fig. 3 ), indicating that the treatment procedures themselves did not contribute significantly to the observed changes in LA innervation. Juvenile treatment did not significantly alter the pattern of innervation in EDL muscles at 9 weeks [normals: $3 \pm 1.4 \%$ (mean percent multiple innervation \pm SEM); oil-treated controls: $0.2 \pm 0.2 \%$; androgen-treated castrates: $1 \pm 1.4 \%$; oil-treated castrates: $2 \pm 0.8 \%, p>0.05$, 1-way ANOVA]. The percentage of multiply innervated muscle fibers was not measured for EDL muscles at 13 weeks.

Number of junctional sites on a fiber. Androgen treatment during synapse elimination induced the formation of closely spaced multiple junctions on some multiply innervated LA fibers (Jordan et al., 1989). Such junctions were also found to persist beyond the end of androgen treatment. At 9 and 13 weeks, LA muscles from JAT castrates contained multiply innervated fibers that possessed either single or multiple junctional sites (Fig. 1), in approximately the same proportions as observed in androgen-treated muscles at 4 weeks (Fig. 3; Jordan et al., 1989). As for multiple junctional sites in androgen-treated muscles at 4 weeks, such multiple junctions in JAT adult muscles were generally less than $10 \mu \mathrm{m}$ apart and were considerably more closely spaced than the multiple junctions occurring on LA fibers in normal adult muscles (which were $45 \mu \mathrm{m}$ apart on average). Furthermore, the multisite innervation in JAT adult LA muscles showed the same sort of heterogeneity as seen in androgen-treated muscles at 4 weeks: Multiple junctions were formed either by different myelinated axons or by unmyelinated preterminal branches from the same axon (Fig. 1), and about a 


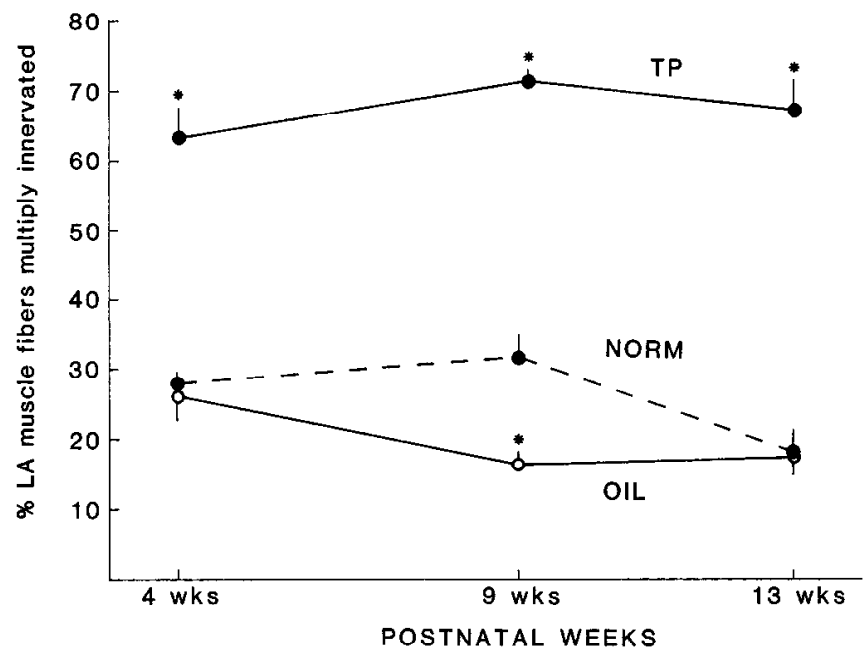

Figure 2. Percentage of multiply innervated fibers in TNBT-stained LA muscles 4, 9, and 13 weeks after birth, derived from counts of the number of motor axons synapsing on individual muscle fibers. Each data point represents the mean ( \pm SEM) of muscles from 4-7 animals per group. The data at 9 and 13 weeks were obtained 4 and 8 weeks after the end of androgen (or oil) treatment, whereas the data at 4 weeks (from Jordan et al., 1989) was obtained during treatment. Multiple innervation persists after the end of juvenile androgen treatment $(T P)$, whereas the secondary phase of synapse loss occurs a month earlier in muscles that lack endogenous gonadal androgen $(O I L)$ than in untreated, normal (NORM) muscles. Asterisks indicate significant differences from normal.

third of the multisite fibers received convergent innervation at one or more sites on the same fiber. Figure 3 presents the percentage of multiply innervated fibers in LA muscles that have either single or multiple junctional sites.

At 9 and 13 weeks, the LA from JAT castrates had significantly more multiply innervated fibers of both types than normal muscles at these ages $(p<0.001$ for single junctional site fibers, $p<0.001$ for multiple junctional site fibers at both adult ages), and this was comparable to the quantitative differences observed at 4 weeks (Fig. 3; Jordan et al., 1989). For JAT LA muscles, the proportions of multiply innervated fibers having either single or multiple junctional sites did not change significantly with age (4-13 weeks, $p>0.05$; Fig. 3).

Number of preterminal branches innervating $L A$ fibers. The increased level of multiple innervation in androgen-treated LA muscles at P28 was also correlated with an increased percentage of LA fibers innervated by axons that branched preterminally (Jordan et al., 1989). Such preterminal branching in adult LA muscle occurs distal to the last segment of myelin and gives rise to multiple unmyelinated branches that innervate the same fiber (Jordan et al., 1988a). Preterminal branching therefore involved short $(<10 \mu \mathrm{m})$ unmyelinated branches from the same parent axon, whereas multiple innervation involved 2 or more myelinated axons (length $\geq 20 \mu \mathrm{m}$ ), possibly from different parent axons. At 9 and 13 weeks, the incidence of LA fibers innervated by preterminal branches in JAT muscles was significantly higher than in normal muscles of the same age $(p<0.05$ at 9 weeks, $p<0.001$ at 13 weeks; Fig. 4), indicating that preterminal branching remained at an enhanced level, despite the lack of androgen. The incidence of preterminal branching in LA muscles from other groups (juvenile oil-treated castrates or sham castrates) was similar to that found in normal LA muscles of

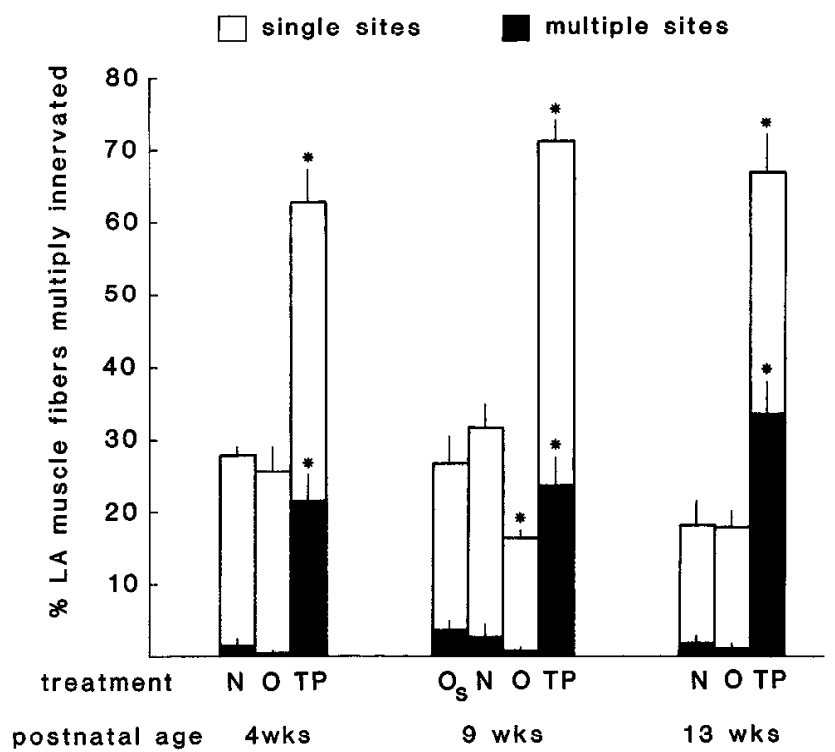

Figure 3. Percentage of multiply innervated LA muscle fibers having singlc or multiple junctional sites. $N$ indicates muscles from normal males; $O_{s}$, muscles from oil-treated sham castrates; $O$, muscles from oil-treated castrates; and $T P$, muscles from androgen-treated castrates. The height of the bar indicates the total mean percentage of multiply innervated fibers. The open part of the bar indicates that portion of multiply innervated fibers having single junctional sites $( \pm$ SEM), and the filled part of the bar indicates that portion of multiply innervated fibers having multiple junctional sites ( \pm SEM). Both types of multiple innervation persist in the LA after the end of juvenile androgen treatment.

the same age ( $p>0.05$ for all comparisons at 9 and 13 weeks). Interestingly, only androgen-treated muscles exhibited an overall increase in the level of preterminal branching between 4 and 13 weeks $(p<0.02)$.

\section{Electrophysiological data}

Although extensive electrophysiological recording from these junctions has not been done, preliminary experiments confirm the effect of androgen in prolonging the period of multiple (polyneuronal) innervation. Adult LA muscles previously treated with androgen contained significantly more polyneuronally innervated fibers than normal adult muscles [18 $\pm 1.1 \%$ (mean \pm SEM) and $2 \pm 1.4 \%$, respectively; $p<0.001$ ]. Figure 5 shows a typical example of a multicomponent EPP indicating that a fiber was polyneuronally innervated. No more than 2 synaptic inputs per fiber were ever detected electrophysiologically.

\section{Pattern of $L A$ muscle fiber growth}

Between 4 and 13 weeks, the pattern of muscle fiber growth depended on whether gonadal androgen was present beyond the end of puberty (after 9 weeks). Although LA fibers in all groups increased in diameter between 4 and 9 weeks $(p<0.001$ for each gruup), only LA fibers in normal muscles (and not castrates) continued to increase in diameter between 9 and 13 weeks ( $p$ $<0.001$, Fig. 6). This result suggests that there are 2 distinct periods of growth for LA muscle fibers, one between 4-9 weeks in which growth of LA fibers can occur independent of androgen and the other between 9-13 weeks in which fiber growth requires androgen.

The effect of JAT on the diameter of LA muscle fibers persisted beyond the end of treatment, since at 9 and 13 weeks, 


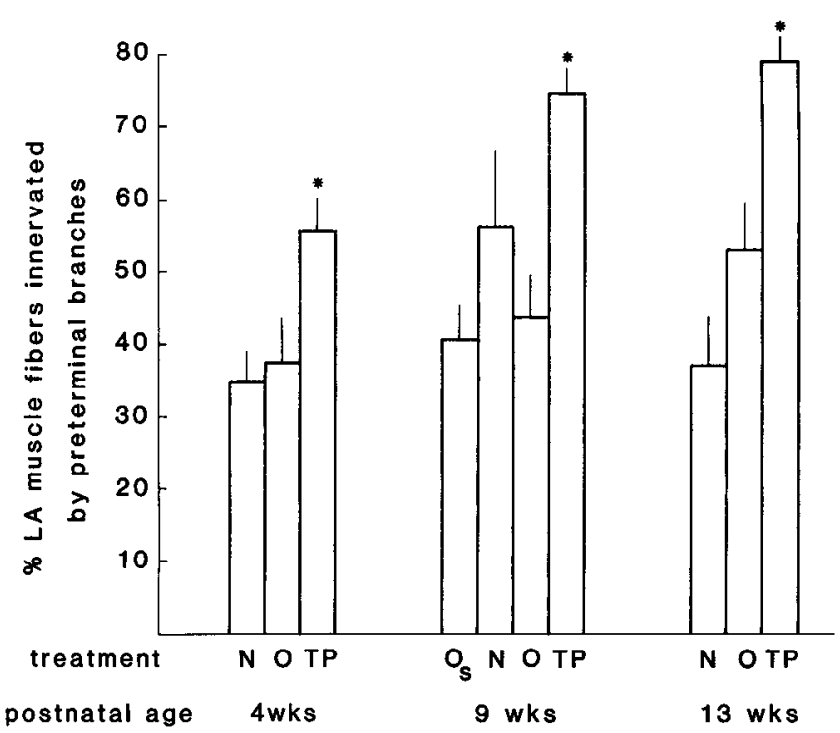

Figure 4. Percentage of LA muscle fibers innervated by unmyelinated preterminal branches from the same axon. Symbols for treatment groups are the same as indicated for Figure 3. The height of the bar represents the mean percentage of fibers innervated by preterminal branches $( \pm$ SEM) based on muscles from 4-7 animals per group. Asterisks indicate significant differences from normal. The increased level of preterminal branching in androgen-treated muscles at 4 weeks (during androgen treatment) remains significantly higher than normal at 9 and 13 weeks, which is after the end of treatment.

LA fibers in JAT muscles were significantly larger than LA fibers in muscles from oil-treated castrates ( $p$ 's $<0.001$; Fig. 6). However, at these adult ages, LA fibers in JAT muscles were significantly smaller than normal $(p<0.03$ and 0.001 , respectively; Fig. 6). Their smaller than normal size probably reflects the lack of adult gonadal androgens, since castration in adulthood reduces the size of LA fibers (Wainman and Shipounoff, 1941; Venable, 1966; Galavazi and Szirman, 1971). Juvenile treatment did not influence the size of EDL fibers at 9 weeks [normals: $41 \pm 0.7 \mu \mathrm{m}$ (mean $\pm \mathrm{SEM}$ ); oil-treated sham castrates: $42 \pm 0.8 \mu \mathrm{m}$; androgen-treated castrates: $44 \pm 1.8 \mu \mathrm{m}$; oiltreated castrates: $39 \pm 0.7 \mu \mathrm{m} ; p>0.05,1$-way ANOVA].

\section{SNB motoneuron number and size}

The number of SNB motoneurons at 9 weeks was the same in all groups, despite differences in hormone treatment during the juvenile period (P7-34) (Fig. 7). Juvenile androgen-treated castrates had the same number of SNB motoncurons as juvenile oil-treated castrates or as late-castrated controls $(p>0.05$ for both comparisons), suggesting that the increased level of multiple innervation in JAT LA muscles is probably not due to an increased number of motoneurons. This finding is consistent with previous work showing that the adult number of SNB motoneurons in female spinal cord is increased by androgen only during a perinatal period that ends before P7 (Breedlove and Arnold, 1983b). However, the size of SNB somata at 9 weeks did depend on the juvenile treatment (Fig. 7). The crosssectional areas of SNB somata in JAT castrates were significantly larger than in juvenile oil-treated castrates $(p<0.02)$. They were also larger than in late-castrated controls, although not significantly $(p>0.05)$. The cross-sectional areas of nuclei of SNB motoneurons at 9 weeks were not significantly influenced by juvenile androgen $\left[180 \pm 17.6 \mu \mathrm{m}^{2}\right.$ (mean $\pm \mathrm{SEM}$ ) for an-

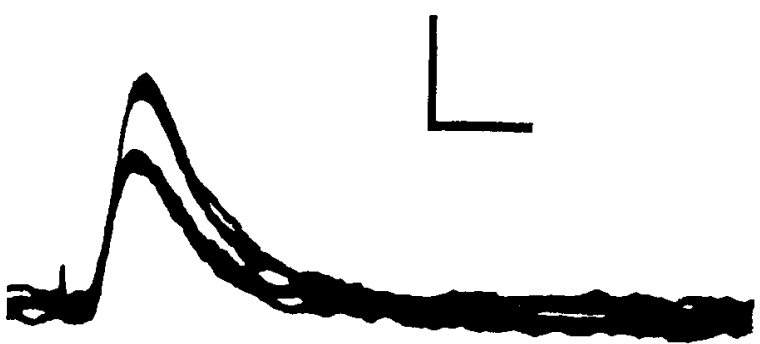

Figure 5. Multiple superimposed oscilloscope traces of intracellularly recorded EPPs from a single LA muscle fiber of a 10 -week-old muscle previously exposed to androgen treatment. As stimulus strength was increased, the amplitude of the EPP also increased in a stepwise fashion, indicating that this particular LA fiber was innervated by at least 2 different motor axons. Such polyncuronally innervated fibers were more frequent in adult LA muscles previously treated with androgen than in normal adult muscles. Vertical calibration bar, $2 \mathrm{mV}$; horizontal calibration bar, $5 \mathrm{msec}$.

drogen-treated castrates compared with $145 \pm 5.0 \mu \mathrm{m}^{2}$ for oiltreated castrates $(p>0.05)$ or compared with $175 \pm 17.6 \mu \mathrm{m}^{2}$ for late-castrated controls $(p>0.05)]$.

\section{Discussion}

The present experiments show that the adult pattern of innervation in the LA, but not in the EDL, is influenced by early androgen exposure. Much of the multiple innervation present in the LA at 2 weeks of age is maintained by androgen treatment during the period of synapse elimination (Pl4-28) (Jordan et al., 1989) and is retained in these muscles for at least 2 months beyond the end of treatment. This result suggests that androgen permanently prevented synapse elimination in the LA.

\section{Alterations in the adult pattern of innervation in the $L A$}

Counting the number of separate TNBT-stained axons contacting individual fibers in LA muscles from 9- and 13-weekold males revealed that while LA muscles from normal and oiltreated castrates continue to experience some degree of synapse loss beyond P28, muscles from JAT castrates do not experience any further loss of synapses. The percentage of multiply innervated LA fibers does not change significantly in androgen-treated muscles between 4 and 13 weeks, despite the fact that muscles after 5 weeks are no longer exposed to gonadal androgen (Fig. 2 ). Since the percentage of multiply innervated fibers is a measure that would not reveal losses in the number of higher-order ( 3 or more) axonal inputs, we also calculated the mean number of axons innervating LA muscle fibers (a measure that is sensitive to overall changes in axon number) in androgen-treated muscles at 4,9 , and 13 weeks. This number also did not change appreciably after the end of androgen treatment $[1.72 \pm 0.06$ ( $\pm \mathrm{SEM}), 1.85 \pm 0.04$, and $1.87 \pm 0.05$, respectively], strongly suggesting that juvenile androgen treatment has rendered the innervation pattern stable and no longer susceptible to further loss.

The processes underlying synapse elimination in the LA are apparently normally active beyond $\mathrm{P} 28$, since there is a continued although modest decline in the level of multiple innervation in the LA of normal males between 9-13 weeks (Fig. 2). This secondary phase of synapse loss occurs a month earlier in muscles from oil-treated castrates, between 4-9 weeks (Fig. 2), who have been deprived of the pubertal surge of androgen that occurs between 4 and 9 weeks after birth (Corpechot et al., 1981). 


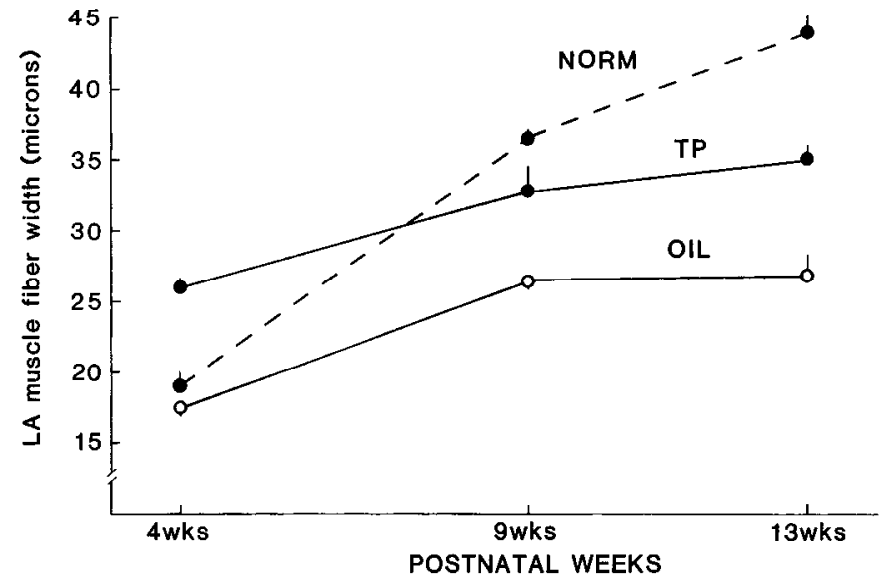

Figure 6. Pattern of LA muscle fiber growth based on measurements of the diameter of individual fibers. Each data point indicates the mean ( \pm SEM) fiber diameter based on muscles from 4-7 animals per group. Labels for treatment groups are defined as in Figure 2. There are 2 distinct periods of LA muscle fiber growth, one between 4-9 weeks in which fiber growth can occur independent of androgen, and the other between 9-13 weeks in which growth requires androgen. The effect of juvenile androgen on LA muscle fiber size persists beyond the the end of treatment, since $T P$ muscles at 9 and 13 weeks contain larger muscle fibers than $O I L$ muscles.

Therefore, this pubertal increase in androgen may normally delay the secondary period of synapse loss. Earlier phases of synapse elimination also appear to begin sooner than normal in LA muscles from oil-treated castrates (Jordan et al., 1989), suggesting that endogenous androgen both before and during puberty play a role in determining the normal time course of this process.

Previous studies have suggested that physical separation of convergent inputs to a cell is a means for mitigating competitive processes that would otherwise lead to the removal of such convergent innervation (Brown et al., 1976; Kuffler et al., 1977; Grinnell et al., 1979; Hume and Purves, 1981). For example, multiple innervation by 2 foreign nerves is preserved in muscle if the innervation occurs at separate sites along the fiber (Kuffler et al., 1977). Similarly, separation of convergent inputs appears to be an important factor ensuring the preservation of multiple innervation in autonomic ganglia (Hume and Purves, 1981), in which different axons innervate single neurons only if they can occupy different regions of the dendritic arbor (Purves and Hume, 1981; Hume and Purves, 1983; Forehand and Purves, 1984). Thus, one might have expected that multiple innervation at single junctional sites would be preferentially eliminated once hormone treatment ended, whereas multiple innervation at separate junctional sites might be more likely to be maintained after treatment. This expectation was not supported by the data. Multiple innervation of both forms are maintained in the LA after the end of androgen treatment (Fig. 3). Moreover, the degree of separation (often only a few microns) between convergent inputs on LA muscle fibers is much less than the amount of separation thought to be required $(>1000 \mu \mathrm{m})$ to maintain multiple innervation in muscle (Brown et al., 1976; Kuffler et al., 1977). Although separation of convergent inputs may well be an important mechanism for ensuring the long-term maintenance and stability of multiple innervation in some systems, it is clearly not a requirement in the LA. Indeed, conclusions
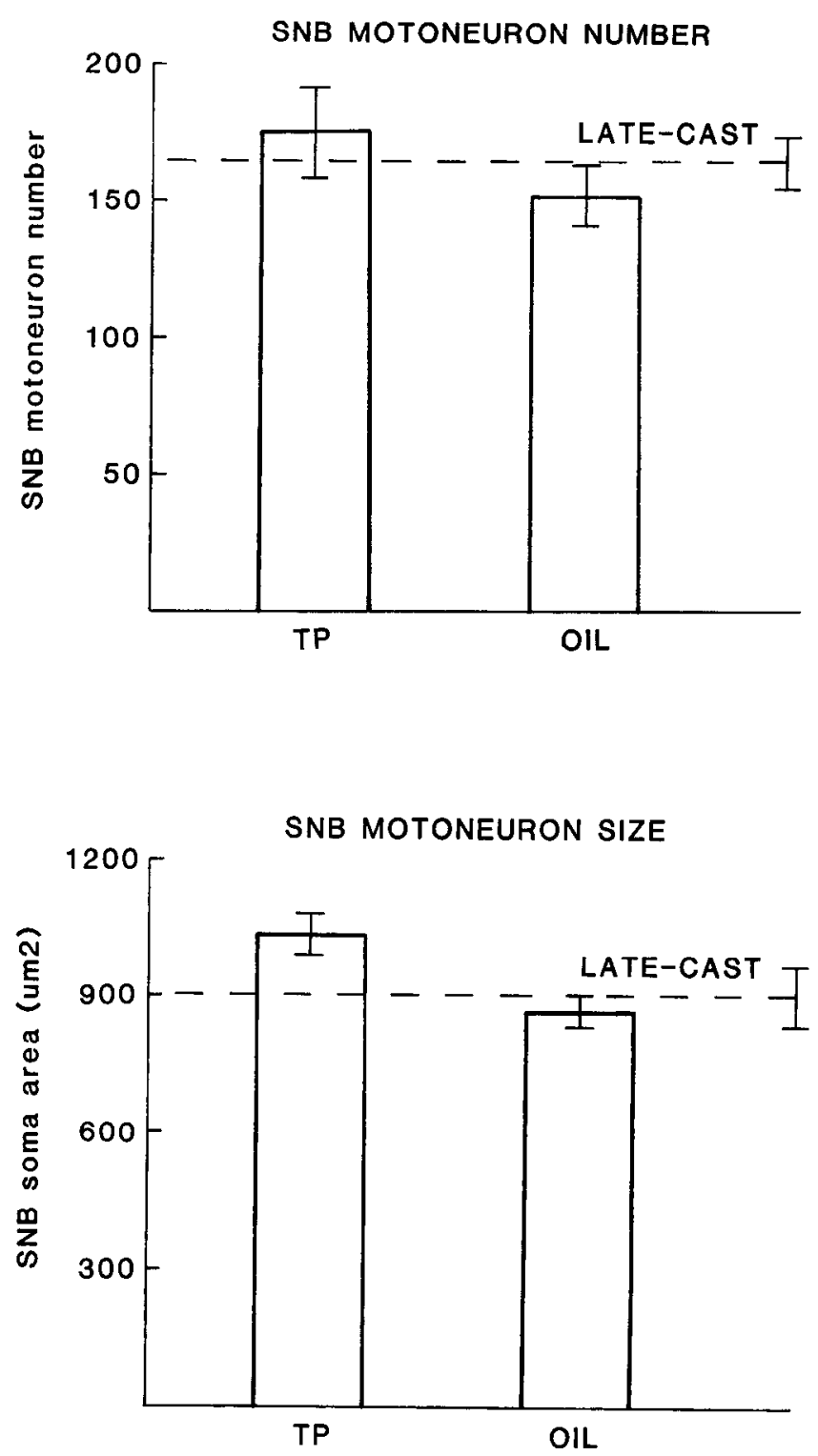

Figure 7. Number and size of SNB motoneurons in lumbar spinal cord of 9-week-old males. The height of the bars indicates the mean number or somatic area of SNB motoneurons in males castrated at P7 and treated with androgen $(T P)$ or oil $(O I L)$ between $\mathrm{P} 7$ and 34 . The dashed line indicates the mean number or somatic area of SNB motoneurons for males that were untreated during the juvenile period (P7-34) and were castrated at P35 (LATE-CAST). Mean estimates ( \pm SEM) based on spinal cords from 4-5 animals per group. At 9 weeks, TP castrates had larger but no more SNB motoneurons than oil-treated castrates.

similar to our own have been made about the spacing of persistent multiple inputs in adult frog sartorius muscle (Werle and Herrera, 1987).

Preliminary electrophysiological recordings revealed significantly more polyneuronal innervation in JAT adult LA muscles $(18 \%)$ than in normal adult LA muscles $(2 \%)$. This finding presumably reflects the fact that androgen prevented the normal loss of polyneuronal innervation during synapse elimination. Although our physiological data corroborates our anatomical data in showing the predicted difference in polyneuronal or multiple innervation, they suggest levels of polyneuronal innervation considerably lower than our anatomical estimates. 
One possible explanation for this discrepancy is that some of the multiple innervation that we observe anatomically actually represents innervation by branches from the same motor axons. However, it seems more likely that the electrophysiological method used (whole nerve stimulation and curare to block muscle twitch) yielded substantial underestimates in the degree of polyneuronal innervation (Herrera, 1984). Polyneuronal inputs to mature muscle fibers tend to produce smaller endplate potentials than the inputs to singly innervated fibers (Taxt et al., 1983; Weakly and Yao, 1983; Trussell and Grinnell, 1985). These small amplitude inputs may have been difficult to detect in the present experiments.

Neuromuscular inactivity produces a similar delay in synapse elimination, but unlike androgen treatment, has not been found to prevent this process irreversibly. Thompson (1985) reported that the loss of multiple innervation in the rat soleus muscle was virtually completely blocked during a 9 day period of inactivity, but once the activity block was removed, synapse elimination occurred outside of the normal period of synapse elimination. This finding suggests that the effects of androgen and inactivity on synapse elimination may be fundamentally different. However, it is important to note that the duration (4 weeks) of androgen treatment in the present experiment was much longer than the duration of the activity block in Thompson's (1985) study. Thus, a longer period of inactivity may have a similar irreversible effect on synapse elimination. Conversely, shorter periods of androgen treatment might only transiently delay synapse elimination, but this seems unlikely. Androgen treatment lasting for only 2 weeks (P7-20) is also effective in producing high levels of multiple innervation in the LA at 9 weeks (Jordan et al., 1988b).

There are several possible mechanisms to explain androgen's long-lasting effect on synapse elimination. Since neuromuscular inactivity prevents synapse elimination (Thompson, 1985), one possibility is that androgen has permanently silenced motor nerve or muscle activity. Although we have not tested this idea directly, this possibility seems unlikely. The LA is part of the SNB neuromuscular system, which mediates penile flip reflexes that are normally active during male copulatory behavior (Sachs, 1982; Hart and Melese-d'Hospital, 1983). Both the penile reflexes and copulation depend on androgen for their activation (Beach and Levinson, 1950; Hart and Haugen, 1968; Hart, 1978; Sachs, 1978). Moreover, the physiological effects of androgen on the LA muscle and its nerve terminals argue that androgen's role is to activate rather than silence the SNB system (Vyskocil and Gutmann, 1977; Souccar et al., 1982a, b). If androgen influences synapse elimination in the LA through an effect on neuromuscular activity, it may be more likely that androgen acts to synchronize rather than block the activity of SNB motoneurons and/or LA muscle fibers. Findings from a number of studies suggest that synchronous activation may be a mechanism for maintaining convergent innervation (Hubel and Wiesel, 1965; Hubel et al., 1977; Stryker and Strickland, 1984). In this regard, it is noteworthy that adult androgen treatment increases the number of morphologically identified gap junctions found between SNB motoneurons (Matsumoto et al., 1988b). It is also possible, however, that androgen influences synapse elimination through a mechanism unrelated to neuromuscular activity, possibly by permanently increasing the amount of muscle-supplied trophic substance for which motor terminals are presumed to compete during synapse elimination (Jansen et al., 1978; Purves, 1986).

\section{Size of SNB motoneurons}

Juvenile androgen treatment produced a small but significant increase in the size, but not number, of SNB motoneurons (Fig. 7). Because of the correlation between the size of a motoneuron and the size of its motor unit (McPhedran et al., 1965; Wuerker et al., 1965; Yao and Weakly, 1986), juvenile androgen treatment probably also increased the size of motor units, which would be consistent with the high number of multiply innervated fibers in JAT LA muscles. However, the effect of androgen on SNB soma size need not correlate with changes in motor unit size or its effects on synapse elimination. For example, normal males and JAT castrates at 9 weeks have LA muscles with very different amounts of multiple innervation (Fig. 2) and, therefore, motor units that probably differ greatly in size. Both, however, have SNB motoneurons with the same mean soma area [1026 $\pm 36 \mu \mathrm{m}^{2}$ ( $\left.\pm \mathrm{SEM}\right)$ vs. $1042 \pm 43 \mu \mathrm{m}^{2}$, respectively]. Moreover, androgen can increase the size of SNB motoneurons outside of the period of synapse elimination (Breedlove and Arnold, 1981; Kurz et al., 1986) without influencing the pattern of innervation in the LA (C. L. Jordan, unpublished observations). Although these findings argue against the possibility that androgen's effect on synapse elimination is through its effect on SNB soma size, they do not exclude the possibility that the androgen-induced increase in SNB soma size that occurs during synapse elimination may have a role in determining the number of motor terminals a motoneuron can maintain. Since SNB motoneurons and the LA muscle have androgen receptors in adulthood (Dionne et al., 1979; Breedlove and Arnold, 1980), each represents a potential site for androgen's effect on synapse elimination and the size of SNB motoneurons.

\section{A possible mechanism for sexual differentiation of the brain}

Over the last 3 decades it has become evident that sex differences in behavior result from structural sex differences in the nervous system (Arnold and Gorski, 1984). One of the earliest reports of a sex difference in brain organization involved differences in the number of synapses (Raisman and Field, 1973). In the preoptic area of the hypothalamus in rats, adult females have more nonstrial inputs onto dendritic spines than do males. Moreover, this sex difference is reversible by manipulating the hormonal environment during the first weeks following birth, but not in adulthood. Other regions of the brain show sex differences in the opposite direction. For example, males have more synapses than females in the ventrolateral-ventromedial nucleus of the hypothalamus and the medial amygdaloid nucleus, and such differences are also detcrmined by gonadal hormones during postnatal development (Nishizuka and Arai, 1981a, b; Matsumoto and Arai, 1981, 1986a, b).

Adult sex differences in the synaptic connectivity of the brain might be produced through a hormonal control of such processes as synaptogenesis, cell death, and/or synapse elimination. Indeed, evidence suggests that both cell death and synaptogenesis can be regulated by gonadal hormones (Matsumoto and Arai, 1979; Nishizuka and Arai, 1982; Breedlove, 1984; DeVoogd et al., 1985; Nordeen et al., 1985; Matsumoto et al., 1988a). The results of the present experiment suggest that gonadal hormones may also alter the adult number of synapses through a regulation of synapse elimination. Since synapse elimination also occurs in the brain (e.g., Crepel et al., 1976; Jackson and Parks, 1982), our results raise the possibility that synapse elimination in other steroid-sensitive parts of the nervous system may be subject to 
a similar regulation by hormones, thereby establishing adult sex differences in the synaptic organization of the brain.

\section{References}

Arai, Y., A. Matsumoto, and M. Nishizuka (1986) Synaptogenesis and neuronal plasticity to gonadal steroids: Implications for the development of sexual dimorphism in the neuroendrocrine brain. Curr. Top. Neuroendocrinol. 7: 291-307.

Arnold, A. P., and R. A. Gorski (1984) Gonadal steroid induction of structural sex differences in the CNS. Annu. Rev. Neurosci. 7: 413442.

Arnold, A. P., and C. L. Jordon (1988) Hormonal organization of neural circuits. In Frontiers of Neuroendocrinology, Vol. 10, I. Martini and W. F. Ganong, eds., pp. 185-213, Raven, New York.

Balice-Gordon, R. J., and W. J. Thompson (1988) Synaptic rearrangements and alterations in motor unit properties in neonatal rat extensor digitorum longus muscle. J. Physiol. (Lond.) 398: 191-210.

Beach, F. A., and G. Levinson (1950) Effects of androgen on the glan penis and mating behavior of castrated male rats. J. Exp. Zool. 114: 159-171.

Bennett, K. L., and J. W. Truman (1985) Steroid-dependent survival of identifiable neurons in cultured ganglia of the moth Manduca sexta. Science 229: 58-60.

Betz, W. J. (1987) Motoneuron death and synapse elimination in vertebrates. In The Vertebrate Neuromuscular Junction, M. M. Salpeter, ed., pp. 117-162, Liss, New York.

Breedlove, S. M. (1984) Androgen forms sexually dimorphic spinal nucleus by saving motoneurons from programmed death. Soc. Neurosci. Abstr. 10:927.

Breedlove, S. M. (1985) Hormonal control of the anatomical specificity of motoneuron-to-muscle innervation in rats. Science 227: 13571359.

Breedlove, S. M., and A. P. Arnold (1980) Hormone accumulation in a sexually dimorphic motor nucleus of the rat spinal cord. Science 210: 564-566.

Breedlove, S. M., and A. P. Arnold (1981) Sexually dimorphic motor nucleus in rat spinal cord: Response to adult hormone manipulation, absence in androgen insensitive rats. Brain Res. 225: 297-307.

Breedlove, S. M., and A. P. Arnold (1983a) Hormonal control of a developing neuromuscular system: I. Complete demasculinization of the male rat spinal nucleus of the bulbocavernosus using the antiandrogen flutamide. J. Neurosci. 3: 417-423.

Breedlove, S. M., and A. P. Arnold (1983b) Hormonal control of a developing neuromuscular system: II. Sensitive periods for the androgen induced masculinization of the rat spinal nucleus of the bulbocavernosus. J. Neurosci. 3: 424-432.

Brown, M. C., J. K. S. Jansen, and D. Van Essen (1976) Polyneuronal innervation of skeletal muscle in new-born rats and its elimination during maturation. J. Physiol. (Lond.) 261: 387-422.

Card, D. (1977) Denervation: Sequence of neuromuscular degenerative changes in rats and the effect of stimulation. Exp. Neurol. 54: 251-265.

Corpechot, C., E. E. Baulicu, and P. Robel (1981) Testosterone, dihydrotestosterone and androstanediols in plasma, testes and prostates of rats during development. Acta Endocrinol. 96: 127-135.

Crepel, F., J. Mariani, and N. Delhaye-Bouchaud (1976) Evidence for a multiple innervation of purkinje cells by climbing fibers in the immature rat cerebellum. J. Neurobiol. 7: 567-578.

DeVoogd, T. J., B. Nixdorf, and F. Nottebohm (1985) Synaptogenesis and changes in synaptic morphology related to acquisition of a new behavior. Brain Res. 329: 304-308.

Dionne, F. T., J. Y. Dube, R. L. Lesage, and R. R. Tremblay (1979) In vivo androgen binding in rat skeletal and perineal muscles. Acta Endocrinol. 91: 362-372.

Fishman, R. B., and S. M. Breedlove (1988) Sexual dimorphism in the developing nervous system. In Handbook of Human Growth and Developmental Biology, E. Meisami and P. S. Timiras, eds., pp. 4557, CRC Press, Boca Raton, FL.

Forehand, C. J., and D. Purves (1984) Regional innervation of rabbit ciliary ganglion cells by the terminals of preganglionic axons. J. Neurosci. 4: $1-12$.

Forger, N. G., and S. M. Breedlove (1987) Seasonal variation in mammalian striated muscle mass and motoneuron morphology. J. Neurobiol. 18: 155-165.
Galavazi, G., and J. A. Szirman (1971) Cytomorphometry of skeletal muscle: The influence of age and testosterone on the rat $M$. levator ani. Z. Zellforsch. 121: 507-530.

Grinnell, A. D., M. S. Letinsky, and M. B. Rheuben (1979) Competitive interaction between foreign nerves innervating frog skeletal muscle. J. Physiol. (Lond.) 289: 241-262.

Gurney, M. (1981) Hormonal control of cell form and number in the zebra finch song system. J. Neurosci. 1: 658-673.

Hart, B. L. (1978) Reflexive mechanisms in copulatory behavior. In Sex and Behavior, T. E. McGill, D. A. Dewsbury, and B. D. Sachs, eds., pp. 205-242, Plenum, New York.

Hart, B. L., and C. M. Haugen (1968) Activation of sexual reflexes in male rats by spinal implantation of testosterone. Physiol. Behav. 3: 735-738.

Hart, B. L., and P. Y. Melese-d'Hospital (1983) Penile mechanisms and the role of the striated penile muscles in penile reflexes. Physiol. Behav. 31: 807-813.

Herrera, A. A. (1984) Polyneuronal innervation and quantal transmitter release in formamide-treated frog sartorius muscles. J. Physiol. (Lond.) 355: 267-280.

Hubel, D. H., and T. N. Wiesel (1965) Binocular interaction in striate cortex of kittens reared with artificial squint. J. Neurophysiol. 28: 1041-1059.

Hubel, D. H., T. N. Wiesel, and S. LeVay (1977) Plasticity of ocular dominance columns in monkey striate cortex. Phil. Trans. R. Soc. London [Biol.] 278: 377-409.

Hume, R. I., and D. Purves (1981) Geometry of neonatal neurones and the regulation of synapse elimination. Nature 293: 469-471.

Hume, R. I., and D. Purves (1983) Apportionment of the terminals from single preganglionic axons to target neurones in the rabbit ciliary ganglion. J. Physiol. (Lond.) 338: 259-275.

Jackson, H., and T. N. Parks (1982) Functional synapse elimination in the developing avian cochlear nucleus with simultaneous reduction in cochlear nerve axon branching. J. Neurosci. 2: 1736-1743.

Jacobson, C. D., V. J. Csernus, J. E. Shryne, and R. A. Gorski (1981) The influence of gonadectomy, androgen exposure, or a gonadal graft in the neonatal rat on the volume of the sexually dimorphic nucleus of the preoptic area. J. Neurosci. 1: 1142-1147.

Jansen, J. K. S., W. Thompson, and D. P. Kuffler (1978) The formation and maintenance of synaptic connections as illustrated by studies of the neuromuscular junction. Prog. Brain Res. 48: 3-18.

Jordan, C. L., M. S. Letinsky, and A. P. Arnold (1988a) Synapse elimination occurs late in the hormone-sensitive levator ani muscle of the rat. J. Neurobiol. 19: 335-356.

Jordan, C. L., M. S. Letinsky, and A. P. Arnold (1988b) Critical period for the androgenic block of neuromuscular synapse elimination. Soc. Neurosci. Abstr. 14: 179.

Jordan, C., M. S. Letinsky, and A. P. Arnold (1989) The role of gonadal hormones in neuromuscular synapse elimination in rats: I. Androgen delays the loss of multiple innervation in the levator ani muscle. J. Neurosci. 9: 229-238.

Keppel, G. (1982) Design and Analysis, Prentice-Hall, Englewood Cliffs, NJ.

Konigsmark, B. W. (1970) Methods for the counting of ncurons. In Contemporary Research Methods in Neuroanatomy, W. J. H. Nauta and S. O. E. Ebbeson, eds., pp. 315-340, Springer-Verlag, New York.

Kuffer, D. P., W. Thompson, and J. K. S. Jansen (1977) The elimination of synapses in multiply-innervated skeletal muscle fibres of the rat: Dependence on distance between end-plates. Brain Res. 138: 353-358.

Kurz, E. M., D. R. Sengelaub, and A. P. Arnold (1986) Androgens regulate dendritic length of sexually dimorphic mammalian motoneurons in adulthood. Science 232: 395-398.

Lee, J. H., C. L. Jordan, and A. P. Arnold (1988) Critical period for androgenic regulation of soma size of sexually dimorphic motoneurons in rat spinal cord. Neurosci. Lett. (in press).

Matsumoto, A., and Y. Arai (1979) Synaptogenic effect of estrogen on the hypothalamic arcuate nucleus of the adult femalc rat. Cell Tissue Res. 198: 427-433.

Matsumoto, A., and Y. Arai (1981) Effect of androgen on sexual differentiation of synaptic organization in the hypothalamic arcuate nucleus: An ontogenetic study. Neuroendocrinology 33: 166-169.

Matsumoto, A., and Y. Arai (1986a) Development of sexual dimorphism in synaptic organization in the ventromedial nucleus of the hypothalamus in rats. Neurosci. Lett. 68: 165-168. 
Matsumoto, A., and Y. Arai (1986b) Male-female difference in synaptic organization of the ventromedial nucleus of the hypothalamus in the rat. Neuroendocrinology 42: 232-236.

Matsumoto, A., P. E. Micevych, and A. P. Arnold (1988a) Androgen regulates synaptic input to motoneurons of adult rat spinal cord. $\mathbf{J}$. Neurosci. 8: 4168-4176.

Matsumoto, A., A. P. Arnold, G. A. Zampighi, and P. E. Micevych (1988b) Androgenic regulation of gap junctions between motoneurons in the rat spinal cord. J. Neurosci. 8: 4177-4183.

McPhedran, A. M., R. B. Wuerker, and E. Henneman (1965) Properties of motor units in a homogeneous red muscle (soleus) of the cat. J. Neurophysiol. 28: 71-84.

Nishizuka, M., and Y. Arai (1981a) Sexual dimorphism in synaptic organization in the amygdala and its dependence on neonatal hormone environment. Brain Res. 212: 31-38.

Nishizuka, M., and Y. Arai (1981b) Organizational action of estrogen on synaptic pattern in the amygdala: Implications for sexual differentiation of the brain. Brain Res. 213: 422-426.

Nishizuka, M., and Y. Arai (1982) Synapse formation in response to estrogen in the medial amygdala developing in the eye. Proc. Natl. Acad. Sci. USA 79: 7024-7026.

Nordeen, E. J., K. W. Nordeen, D. R. Sengelaub, and A. P. Arnold (1985) Androgens prevent normally occurring cell death in a sexually dimorphic spinal nucleus. Science 229: 671-673.

Nordeen, E. J., K. W. Nordeen, and A. P. Arnold (1987) Sexual differentiation of androgen accumulation in the zebra finch brain through selective cell loss and addition. J. Comp. Neurol. 259: 393-399.

Purves, D. (1986) The trophic theory of neural connections. TINS 9 486-489.

Purves, D., and R. I. Hume (1981) The relation of postsynaptic geometry to the number of presynaptic axons that innervate autonomic ganglion cells. J. Neurosci. $1: 441-452$.

Purves, D., and J. W. Lichtman (1980) Elimination of synapses in the developing nervous system. Science 210:153-157.

Raisman, G., and P. M. Field (1973) Sexual dimorphism in the neuropil of the preoptic area of the rat and its dependence on neonatal androgen. Brain Res. 54: 1-29.

Redfern, P. A. (1970) Neuromuscular transmission in new-born rats J. Physiol. (Lond.) 209: 701-709.

Riley, D. A. (1977) Spontaneous elimination of nerve terminals from the endplates of developing skeletal myofibers. Brain Res. 134: 279285.

Riley, D. A. (1981) Ultrastructural evidence for axon retraction during the spontaneous elimination of polyneuronal innervation of the rat soleus muscle. J. Neurocytol. 10: 425-440.

Sachs, B. D. (1978) Conceptual and neural mechanisms of masculine copulatory behavior. In Sex and Behavior, T. E. McGill, D. A. Dewsbury, and B. D. Sachs, eds., pp. 267-295, Plenum, New York.

Sachs, B. D. (1982) Role of penile muscles in penile reflexes, copulation, and induction of pregnancy in rats. J. Reprod. Fertil. 66:433443.
Sassoon, D., N. Segil, and D. B. Kelley (1986) Androgen-induced myogenesis and chondrogenesis in the larynx of Xenopus laevis. Dev. Biol. 113:135-140.

Schroder, H. D. (1980) Organization of the motoneurons innervating the pelvic muscles of the rat. J. Comp. Neurol. 192: 567-587.

Souccar, C., A. J. Lapa, and J. R. DoValle (1982a) Influence of castration on the electrical excitability and contraction properties of the rat levator ani muscle. Fxp. Neurol. 75: 576-588.

Souccar, C., A. J. Lapa, and J. R. DoValle (1982b) The influence of testosterone on neuromuscular transmission in hormone-sensitive mammalian skeletal muscles. Muscle Nerve 5: 232-237.

Stryker, M. P., and S. L. Strickland (1984) Physiological segregation of ocular dominance columns depends on the pattern of afferent electrical activity. Invest. Ophthalmol. Vis. Sci. (Suppl.) 25: 278.

Taxt, T., R. Ding, and J. K. S. Jansen (1983) A note on the elimination of polyneuronal innervation of skeletal muscles in neonatal rats. Acta Physiol. Scand. 117: 557-560.

Thompson, W. (1985) Activity and synapse elimination at the neuromuscular junction. Cell. Mol. Neurobiol. 5: 167-182.

Thompson, W. J. (1986) Changes in the innervation of mammalian skeletal muscle fibers during postnatal development. TINS 9: 25-28.

Toran-Allerand, C. D. (1976) Sex steroids and development of the newborn mouse hypothalamus and preoptic area in vitro: Implications for sexual differentiation. Brain Res. 106: 407-412.

Trussell, L. O., and A. D. Grinnell (1985) The regulation of synaptic strength within motor units of the frog cutaneous pectoris muscle. $\mathrm{J}$. Neurosci. 1: 243-254.

Van Essen, D. C. (1982) Neuromuscular synapse elimination. In Neuronal Development, N. C. Spitzer, ed., pp. 333-376, Plenum, New York.

Venable, J. H. (1966) Morphology of the cells of normal, testosteronedeprived, and testosterone-stimulated levator ani muscles. Am. J. Anat. 119: 271-302

Vyskocil, F., and E. Gutmann (1977) Anabolic effects of testosterone on the levator ani muscle of the rat. Pfluegers Arch. 371: 3-8.

Wainman, P., and G. C. Shipounoff (1941) The effects of castration and testosterone propionate on the striated perineal musculature in the rat. Endocrinology 29: 975-978.

Weakly, J. N., and Y.-M. M. Yao (1983) Synaptic efficacy at singlyand dually-innervated neuromuscular junctions in the frog, Rana pipiens. Brain Res. 273: 319-323.

Werle, M. J., and A. A. Herrera (1987) Synaptic competition and the persistence of polyneuronal innervation at frog neuromuscular junctions. J. Neurobiol. 18: 375-389.

Wuerker, R. B., A. M. McPhedran, and E. Henneman (1965) Properties of motor units in a heterogeneous pale muscle $(\mathrm{m}$. gastrocnemius) of the cat. J. Neurophysiol. 28: 85-99.

Yao, Y.-M. M., and J. N. Weakly (1986) Differences in transmitter release and number of nerve terminals per motoneuron between two frog muscles. J. Neurosci. 6: 498-506. 